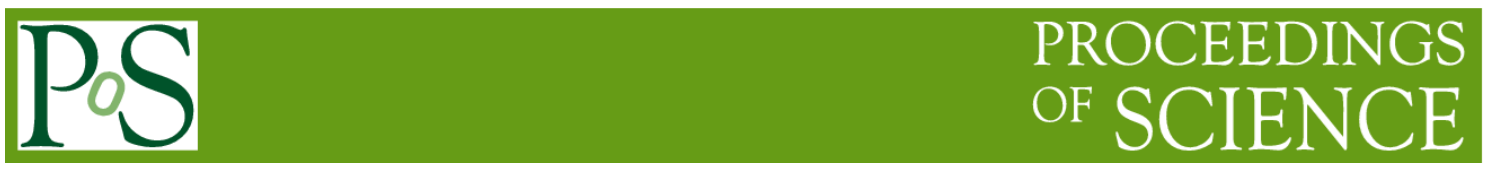

\title{
Galactic and Local Universe VLBI With MeerKAT
}

\author{
Gordon MacLeod ${ }^{1}$ \\ Hartebeesthoek Radio Astronomy Observatory \\ Krugersdorp, South Africa \\ E-mail: gordehartrao.ac.za \\ Michael Bietenholz \\ Hartebeesthoek Radio Astronomy Observatory \\ Krugersdorp, South Africa \\ And York University, Canada \\ E-mail: michaelehartrao.ac.za
}

MeerKAT will be the most sensitive radio telescope in the Southern hemisphere, and one of only very few VLBI-capable telescopes in the south. A VLBI-capable MeerKAT would considerably increase the sensitivity, as well as effectively increase the resolution and imaging capability of an available VLBI array for southern-sky observations. There is a very wide range of science areas where this capability has great potential for stunning science returns, including transients, pulsars, masers, the structure of our own Galaxy, extragalactic supernovae, gamma-ray bursts, active galactic nuclei (AGN), and the role AGNs play in galaxy formation. Many of the MeerKAT Large Survey Projects will benefit from or even require some VLBI followup. It is clear therefore that MeerKAT should be made VLBI-capable to take advantage of these opportunities.

MeerKAT Science: On the Pathway to the SKA,

25-27 May, 2016,

Stellenbosch, South Africa

\section{${ }^{1}$ Gordon MacLeod}

(C) Copyright owned by the author(s) under the terms of the Creative Commons 


\section{Introduction}

Very Long Baseline Interferometry (VLBI) has a long list of stunning science returns and will continue to be important in the era of MeerKAT (the South African SKA precursor), and beyond in the era of the Square Kilometre Array, SKA (e.g. see [1]). South Africa already has a long history with VLBI [2]; the addition of a VLBI-capable MeerKAT will extend this long into the future.

MeerKAT will be the most sensitive radio telescope in the southern hemisphere, and adding it to existing VLBI networks will bring a very significant gain in sensitivity as well as effectively increase the resolution and imaging capability for any targets in the southern celestial hemisphere. The major leap in VLBI capability provided by MeerKAT can address a very large range of exciting science goals, including transients (including fast radio bursts), pulsars (including the detection of gravitational waves from ensembles of pulsars), masers, the structure of our own Galaxy, extragalactic supernovae, gamma-ray bursts, active galactic nuclei (AGN) and their role in galaxy formation.

The southern terrestrial hemisphere, and Africa in particular, are poorly served with very few large-diameter VLBI-capable radio telescopes, with only those in Africa at the Hartebeesthoek Radio Astronomy Observatory (HartRAO; South Africa), which are of modest sensitivity. MeerKAT, which will have over 17 times the effective collecting area of HartRAO, will add greatly to the sensitivity of any VLBI operations in the south, whether it be with the European VLBI Network $(\mathrm{EVN})^{2}$, the Australian Long Baseline Array (LBA) ${ }^{3}$, an array combining elements of both, or even future operations with the African VLBI Network (AVN; see [3]). The long baselines between the MeerKAT and the European or Australian antennas will provide both high sensitivity and high resolution, and the intermediate baselines with HartRAO (and in future, the AVN) will provide the Fourier-plane or $u v$-plane coverage necessary for reliable imaging and allow for robust calibration.

MeerKAT is an interferometer, and it will have 64 dishes of $13.5-\mathrm{m}$ diameter when completed. It will have baseline lengths up to $8 \mathrm{~km}$ and arc-second resolution. For VLBI use the MeerKAT array, either in its entirety or partly, would be phased up and used effectively as a single dish.

\section{MeerKAT Large Survey Projects and VLBI}

MeerKAT will spend a significant fraction of its observing time on ten Large Survey Projects (LSPs), which will aim to further our understanding of everything from the formation of galaxies to the structure of our own Galaxy. Milli-arcsecond (mas) resolution VLBI observations will be an important complement to the arc-second resolution achieved with MeerKAT. In particular, a number of the MeerKAT LSPs will benefit or even require some mas-resolution observations, and therefore benefit from a VLBI-capable MeerKAT.

The MIGHTEE survey is one of the MeerKAT LSPs [4] and comprises a very deep continuum survey. It will investigate the evolution of the radio source population from $0<z<6$, which will address, among other questions, the interplay between star formation and active galactic nuclei: do AGN trigger star or quench star formation? An entire starburst galaxy as well as

\footnotetext{
${ }^{2}$ See http://www.evlbi.org

${ }^{3}$ See http://www.atnf.au/vlbi
} 
its possible AGN may all fit within the MeerKAT beam of a few arc-seconds even for sources at $z<1$; therefore VLBI observations would be required to distinguish the two.

The MeerKAT LSP ThunderKAT [5] will use both dedicated time and commensal observations to search for dynamic and explosive transient radio sources, taking advantage of MeerKAT's large field of view and sensitivity. Transients by their nature must be physically small, and follow-up VLBI observations of any detected will be essential to their understanding. VLBI can determine a very precise position of the transient and, more importantly, measure the proper motion and/or expansion speed directly. For example, novae have typical expansion speeds of $5,000 \mathrm{~km} \mathrm{~s}^{-1}$, resolvable in a few days at the mas-resolution of VLBI. Similarly, normal supernovae with $v \sim 20,000 \mathrm{~km} \mathrm{~s}^{-1}$, would be resolvable in about a year at $10 \mathrm{Mpc}$, while relativistic supernovae or gamma-ray bursts (GRBs) expanding at nearly the speed of light are resolvable within one year out to $100 \mathrm{Mpc}$.

The MeerKAT LSP Trapum [6] is designed to search for, and investigate, new and exotic pulsars, and other transients. It will be of great interest to measure proper motions and parallaxes of these pulsars through VLBI observations. Observations of a large sample of pulsars are required to detect gravitational waves through pulsars. Pulsars are typically faint and steep spectrum radio sources, therefore both high sensitivity and high resolution observations are required at low radio frequencies. MeerKAT, operating in the 1-2 GHz band, will be essential for VLBI networks to complete out such observations.

The LSP MeerGAL [7] is a high-frequency survey of the southern Galactic plane, which will detect many new inverted or flat spectrum Galactic sources. Complimentary VLBI observations will be important in efforts to distinguish between background AGNs and compact Galactic sources. These VLBI observations will provide parallax and proper motion measurements of the newly discovered Galactic sources.

The MeerKAT LSP MESMER [8] is a survey of highly redshifted molecular emission mostly targeting CO 115-GHz emission at redshifts of $z>7$. However, MESMER may also detect water masers in gravitationally-lensed systems at lower redshifts of $0.5<z<1.2$ [9]; VLBI observations of these sources can provide direct geometric distances. A direct distance determination was made for NGC 4258 was made on the basis of such VLBI observations [10].

\section{Science with MeerKAT VLBI}

In addition to the VLBI observations which directly contribute to the MeerKAT LSPs there is a wide range of other science requiring VLBI, which would benefit greatly from the boost in sensitivity, resolution and imaging capability provided by adding MeerKAT to the VLBI network.

High resolution observations of transients are important not only for those detected by MeerKAT itself, but also for transients detected by other instruments, e.g. the Australian SKA pathfinder (ASKAP; see [11]) or LOFAR (see e.g. [12]). Similarly, pulsar astrometry is important for pulsars detected outside the TRAPUM LSP. Obtaining precise astrometry and proper motions of a large fraction of the Galactic pulsar population would enable interesting studies. In particular, the prospect of finding a pulsar near to the Galactic centre is exciting, as a number of relativistic effects could be directly measured [14] [15]. Although many neutron stars are expected in the vicinity of the Galactic centre, none have as yet been discovered [13]. If one is discovered it would almost certainly be faint, and thus the most sensitive VLBI array possible 
would be required to measure its proper motion. Finally, VLBI measurements of pulsars, radio stars, and AGNs represent the best opportunity to tie the Gaia celestial reference frame to the existing VLBI-based International Celestial Reference Frame [15].

It might also be possible to detect exoplanets in the radio using VLBI astrometry, in particular around $\mathrm{M}$ and $\mathrm{L}$ dwarf stars, for which optical planet-finding techniques do not work well [16] [17][18]. Astrometry with VLBI holds the exciting prospect of determining the orbit of a planet emitting radio wavelength radiation such as Jupiter.

Astrometry using VLBI will be important in the determination of the structure of our own Galaxy. Astrometry using VLBI can determine parallaxes and hence distances as far out as the Galactic centre. Since much more of the Galaxy is visible from the south, MeerKAT will be a key element in obtaining VLBI parallaxes of faint southern sources. We have already mentioned pulsar astrometry above. However, pulsars are not good tracers of stellar mass content of the Galaxy. By contrast, masers associated with young high-mass stars are excellent tracers, and the determination of precise distances and proper motions will enable us to more accurately map the mass distribution in the Galaxy. Such maser observations have, for example, yielded the first direct measurement of the pitch angles of the arms of the Galaxy [19] and mapping of the northern part of the Galaxy continues [20]. More work is required, in particular on the large part of the Galaxy only accessible from the South, and it is here the MeerKAT will be instrumental.

\section{Impact of MeetKAT on VLBI Capabilities}

MeerKAT will present a very significant addition to the global VLBI capability. It has a large collecting area, and adds very long baselines, and thus high resolution, to the EVN or the Australian LBA. We show examples of the $u v$-plane coverage obtained when using MeerKAT with the EVN and LBA in Figure 1. It could, of course, also be used as an element in ad-hoc arrays with other antennas world-wide such as those of the National Radio Astronomy Observatory in the United States or those in Asia not in the EVN, or primarily geodetic antennas such as that at O'Higgins, Antarctica or TIGO, Chile. MeerKAT will be capable of operating in four of the most commonly used VLBI frequency bands: $1.4 \mathrm{GHz}, 1.7 \mathrm{GHz}$ and 8.4 and $14 \mathrm{GHz}$. A trans-continental VLBI array at $14 \mathrm{GHz}$ with MeerKAT will be able to achieve resolutions smaller than 0.5 mas with high sensitivity. We note that the addition of a 5-8 GHz capability for MeerKAT would be highly desirable for VLBI, both for the $5 \mathrm{GHz}$ continuum and the $6.7 \mathrm{GHz}$ methanol-maser observations. Adding MeerKAT to the Australian LBA will produce an increase in resolution by a factor of $\sim 3$ for sources at far southern declinations (note that sources near the south Celestial pole are not visible to HartRAO due to its equatorial mount). Using MeerKAT with the EVN will greatly increase the north-south resolution for sources at low and southern declinations. In particular, the use of MeerKAT to VLBI arrays will enable higher sensitivity and/or resolution for a significant portion of our own Galaxy.

For the purposes of this discussion, we will assume that the central core of MeerKAT, consisting of $\sim 45$ dishes, can be phased up for VLBI observations. Once phased up, this core will represent a collecting area equivalent that of a $\sim 90 \mathrm{~m}$ diameter dish. Phasing up all 64 dishes, out to baselines of $\sim 8 \mathrm{~km}$, will be equivalent to a $\sim 108 \mathrm{~m}$ dish, comparable in collecting area to the largest fully steerable dishes in existence and to the full Jansky VLA. Of course, smaller sub-arrays of MeerKAT dishes might also be used for some purposes. A benefit in addition to the increased sensitivity of adding MeerKAT to any VLBI experiment which also includes Har- 
tRAO is that when both MeerKAT and HartRAO are used, it will greatly improve the robustness of calibration, which is particularly problematic with isolated VLBI antennas [21].
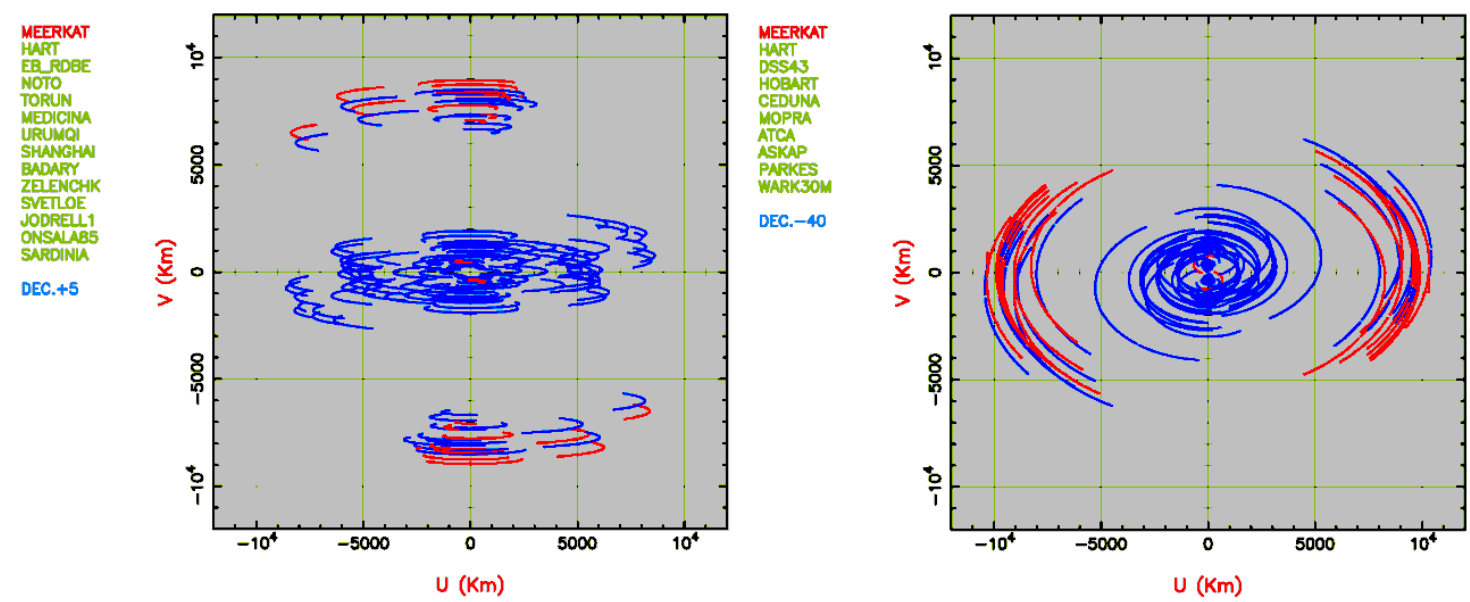

Figure 1: Plots showing the increase in the $u v$-plane coverage obtained when adding MeerKAT, to the EVN (left) and to the Australian LBA (right). The antennas are listed in the left of each panel, with the source declinations below in blue. Our examples show $u v$-coverage for sources at decl. $+5^{\circ}$; observed with the EVN, and at $-40^{\circ}$; with the LBA, respectively. The baselines involving MeerKAT are shown in red, and the remainder in blue. The high sensitivity of MeerKAT, about 10 times more sensitive than HartRAO, makes baselines involving MeerKAT especially valuable, and the presence of a relatively short baseline between MeerKAT and HartRAO adds greatly to the robustness of calibration.

The possibility of MeerKAT having multiple phased-array beams available opens up exciting possibilities for cutting-edge VLBI techniques such as wide-field VLBI and in-beam phase referencing. In particular, it would be possible to divide MeerKAT into several sub-arrays, each pointing in different directions, allowing a multi-beam VLBI capability (i.e. a cluster). The "cluster-cluster" or multi-view VLBI technique [22] offers significant advantages for astrometry and extremely wide field mapping. Candidate cluster sites for joint observations with MeerKAT are HartRAO, ATCA, ASKAP, Hobart, and Parkes in the southern hemisphere, and Jodrell Bank, Westerbork, Yebes, the GMRT and the EVLA in the Northern Hemisphere.

\section{Conclusions}

MeerKAT will be a tremendous asset to the international VLBI community. A VLBIcapable MeerKAT would considerably increase the sensitivity, as well as effectively increase the resolution and imaging capability of the available VLBI array for southern-sky observations. Among the areas of interest where this capability has great potential for stunning science returns are: transients, pulsars, masers, the structure of our own Galaxy, extragalactic supernovae \& gamma-ray bursts, and active galactic nuclei (AGN) \& their role in galaxy formation. Many of the MeerKAT LSPs will benefit from or even require some VLBI follow-up. There is little doubt that MeerKAT should be made VLBI-capable to take advantage of these opportunities.

\section{References}

[1] H. J. van Langevelde 2013, arXiv eprints, 1301.1060. 
[2] G. D. Nicolson 1995, Ap\&SS, 230, 329.

[3] M. J. Gaylard, M. F. Bietenholz, L. Combrinck, R. S. Booth, S. J. Buchner, et al. 2011, Proceedings of the SAIP 2011, the $56^{\text {th }}$ Ann. Conf. of the South African Institute of Physics, ed. I Basson and A. E. Botha (University of Pretoria), p. 473.

[4] M. Jarvis, this volume.

[5] R. Fender \& P. Woudt, this volume.

[6] B. Stappers \& M. Kramer, this volume.

[7] M. Thompson, this volume.

[8] I. Heywood, this volume.

[9] C. M. V. Impellizzeri, J. P. McKean, P. Castangia, A. L. Roy, C. Henkel, A. Brunthaler, \& O. Wucknitz 2008, Nature, 456, 927, 0901.1132.

[10] M. J. Reid, J. A. Braatz, J. J. Condon, L. J. Greenhill, C. Henkel, \& K. Y. Lo 2009, ApJ, 695, 287.

[11] G. Hobbs, I. Heywood, M. E. Bell, M. Kerr, A. Rowlinson, et al. 2016, MNRAS, 456, 3948.

[12] T. Coenen, J. van Leeuwen, J. W. T. Hessels, B. W. Stappers, V. I. Kondratiev et al. 2014, A\&A, 570, 60.

[13] F. Zhang, Y. Lu \& Q. Yu 2014, ApJ, 784, 106.

[14] K. Liu, N. Wex, M. Kramer, J. M. Cordes, \& T. J. W. Lazio 2012, ApJ, 747, 1.

[15] R. N. Manchester, G. B. Hobbs, A. Teoh, \& M. Hobbs 2005, AJ, 129, 1993.

[16] G. C. Bower, A. Bolatto, E. Ford, P. Kalas, \& J. Ulvestad 2007, arXiv eprints, 0704.0238.

[17] J. Forbrich \& E. Berger 2009, ApJL, 706, L205.

[18] J. Forbrich, T. J. Dupuy, M. J. Reid, E. Berger, A. Rizzuto, et al. 2016, ApJ, 827, 22.

[19] M. J. Reid, K. M. Menten, X. W. Zheng, et al. 2009, ApJ, 700, 137.

[20] M. J. Reid, K. M. Menten, A. Brunthaler, X. W. Zheng, T. M. Dame, et al. 2014, ApJ, 783, 130.

[21] N. Kawaguchi, W. Jiang, \& Z.-Q. Shen 2015, PASJ, 67, 112.

[22] M. J. Rioja, R. Dobson, J. Reynolds, \& C. Phillips 2010, Proceedings of ISKAF2010 Science Meeting, "Multi-view VLBI with SKA Pathfinders", published online at http://pos.sissa.it/cgibin/reader/conf.cgi?confid=112, p. 78. 